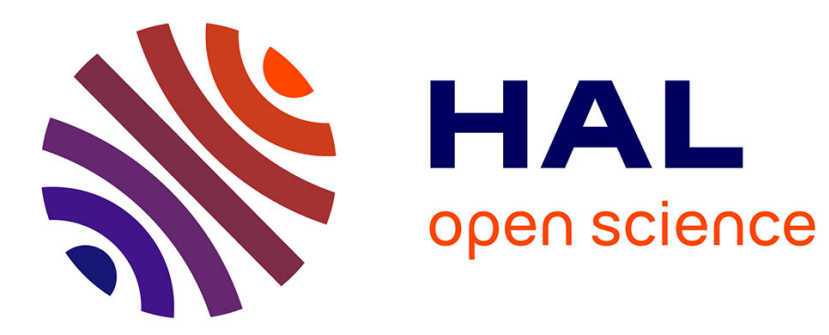

\title{
Impact of the Dipole Tilt Angle on the Ionospheric Plasma as Modeled with IPIM
}

\author{
Aurélie Marchaudon, P -l Blelly
}

\section{To cite this version:}

Aurélie Marchaudon, P -l Blelly. Impact of the Dipole Tilt Angle on the Ionospheric Plasma as Modeled with IPIM. Journal of Geophysical Research Space Physics, 2020, 125, 10.1029/2019ja027672 . hal02990127

\section{HAL Id: hal-02990127 \\ https://hal.science/hal-02990127}

Submitted on 5 Nov 2020

HAL is a multi-disciplinary open access archive for the deposit and dissemination of scientific research documents, whether they are published or not. The documents may come from teaching and research institutions in France or abroad, or from public or private research centers.
L'archive ouverte pluridisciplinaire HAL, est destinée au dépôt et à la diffusion de documents scientifiques de niveau recherche, publiés ou non, émanant des établissements d'enseignement et de recherche français ou étrangers, des laboratoires publics ou privés. 


\section{JGR Space Physics}

\section{RESEARCH ARTICLE \\ 10.1029/2019JA027672 \\ Impact of the Dipole Tilt Angle on the Ionospheric Plasma as Modeled with IPIM}

Key Points:

- Combined corotation and convection in the plasmasphere causes nonuniform drift of the dipole tilt projection in the flux tube plane

- Dipole tilt projection's variation causes variable solar illumination along the tube and day-to-day variation in plasma distribution

- Even for very quiet conditions no equilibrium can be reached in the plasmasphere due to day-to-day variation in the tube plasma distribution

Correspondence to:

A. Marchaudon,

aurelie.marchaudon@irap.omp.eu

Citation:

Marchaudon, A., \& Blelly, P.-L. (2020) Impact of the dipole tilt angle on the ionospheric plasma as modeled with, IPIM. Journal of Geophysical Research: Space Physics, 125, e2019JA027672.

https://doi.org/10.1029/2019JA027672

Received 22 NOV 2019

Accepted 7 MAY 2020

Accepted article online 15 MAY 2020

(C)2020. American Geophysical Union. All Rights Reserved.

\author{
A. Marchaudon ${ }^{1}$ (D) and P.-L. Blelly ${ }^{1}$ (D) \\ ${ }^{1}$ Institut de Recherche en Astrophysique et Planétologie, Université de Toulouse, CNRS, CNES, Toulouse, France
}

\begin{abstract}
We ran the IRAP Plasmasphere Ionosphere Model (IPIM) for several flux tubes at different distances from Earth for very quiet conditions, in order to model the convection and corotation transport of closed flux tubes in the plasmasphere for tilted/eccentric dipolar magnetic field configuration and for solstice and equinox conditions. For each simulation, the model was run for 30 days in order to study the long-term evolution of the plasmasphere-ionosphere system. The goal is to study the combined effect of corotation and convection, which causes eventually a lag of the flux tube motion with respect to the Earth. This combination of nonuniform rotation and subcorotation in average becomes important especially in the outer plasmasphere and causes nonuniform drift of the dipole tilt projection in the plane of the flux tube with time, changing drastically the plasma distribution along this flux tube. In the classical representation of the plasmasphere, the ionosphere only depends on angular Magnetic Local Time (MLT) sector. We show that due to the tilt effect, solar illumination along the flux tube and field-aligned transport varies from one day to the other and causes also a day-to-day variation in the plasma distribution along the tube. This effect implies that a stable representation of the plasmasphere in MLT during quiet conditions is an erroneous view, and no real dynamic equilibrium can be reached, in particular close to the stagnation point.
\end{abstract}

\section{Introduction}

It is well-known that the plasmasphere region is shaped by the competition between corotation near Earth and magnetospheric convection at further distance giving its well-known tear drop pattern (Brice, 1967; Nishida, 1966). However, this static vision is clearly oversimplified, and the need to take into account large scale variations of the magnetospheric convection is essential. Thus, convection intensifications will mainly cause plasmasphere erosion by the depletion of flux tubes, on initially closed streamlines around the Earth, which are then suddenly located on opened sunward convective streamlines. When convection reduces, depleted flux tubes will eventually be enclosed inside the corotation dominated region, allowing them to be refilled by plasma diffusion originating from the ionosphere. While this chain of processes is relatively well understood qualitatively, large unknowns remain especially about the refilling temporal scale for the flux tubes, which is slower than expected in physical simulations and for which bad constraint of thermal conductivity, electron heating by suprathermal electrons and the role of the neutral atmosphere density and the properties of the exobase have been explored (e.g. Comfort et al., 1995; Denton et al., 2014; Horwitz et al., 1990; Liemohn et al., 1997; Reynolds et al., 2003; Tu et al., 2003). Finally, other properties still need to be better understood such as the possibility of a two-stage process depending on the plasma concentration already present inside the tube with faster refilling in the later stage (Lawrence et al., 1999; Su et al., 2001; Thomsen et al., 1998) and the capacity for the flux tube to reach concentration saturation (Reynolds et al., 2003).

Moreover, results from the IMAGE spacecraft, the Cluster and the Van Allen missions have shown that the plasmasphere region is even more complex than expected about 20 years ago. For example, the imager experiments onboard IMAGE have provided instantaneous 2-D views of the complete plasmasphere, showing a complex shape with some indentation (e.g., void and notch) or extension (e.g., shoulder and plume) highlighting the complex dynamics of this region even during quiet periods (Sandel et al., 2001, 2003). In parallel, azimuthal and radial lags of the plasmasphere corotation have also been detected near Earth in the equatorial plane, below 3 Earth's Radii $\left(R_{E}\right)$ (e.g. Burch et al., 2004; Galvan et al., 2010; Sandel et al., 2003), which was rather unexpected at the time. The ionospheric dynamo disturbance through modulation of thermospheric winds has been suggested to explain the azimuthal lag (Burch et al., 2004), but as this lag has 
been recently observed at all latitudes and longitudes, global subcorotation of the thermosphere is suspected (Lejosne \& Mozer, 2017). Increase of magnetic activity on the nightside responsible for subauroral electric drift perturbations could also explain part of the corotation lag observations (Lejosne \& Mozer, 2018).

Finally, a few statistical studies have tried to unveil possible longitudinal and seasonal variations of the plasmasphere electron density content using whistler observations below $3 R_{E}$. Unexpected annual variations of the plasmaspheric equatorial electron density have early been reported (e.g., Clilverd et al., 1991) showing systematically higher electron density during December solstice than during June solstice over America despite one plasmaspheric field line footprint always being in the summer hemisphere. Extensions of this study have shown no such variation over Europe and reversed variation over Asia, which confirmed the role of the Earth's magnetic dipole tilt in this effect (Clilverd et al., 2007; Richards et al., 2000). Indeed, the asymmetric electron content of the plasmasphere between solstices can be explained by the offset between geographic and geomagnetic latitudes; the solstice plasmasphere density being lower for field lines with summer footprint close to the geographic equator, spending more time in darkness. Such effects disappear beyond 4.5 $R_{E}$, where flux tubes are no longer expected to be in diffusive equilibrium (Menk et al., 2012). These annual variations do not seem to be caused by the thermospheric winds but more likely by asymmetric heating of the plasmasphere by photoelectrons (Richards et al., 2000).

While it is possible from observations to unveil the annual and longitudinal variations of the plasmasphere caused by Earth's dipole tilt, it is more difficult to look at local time variations with respect to this dipole tilt and how they possibly evolve with season. Plasmasphere-ionosphere simulations can partly overcome this problem, in following flux tubes during the course of their rotation around the Earth.

The main purpose of this paper is thus to present such a study where flux tubes are modeled with the IRAP Plasmasphere Ionosphere Model (IPIM) (Marchaudon \& Blelly, 2015) at different distances from the Earth. The simulations are run over long time periods (about 30 days), under steady electrodynamic conditions. The electric field results from cross-potential in the 3-15 MLT direction, which is combined with the corotation and causes the tear drop shape visible in Figure 1 (redrawn from Marchaudon \& Blelly, 2015), which is described in the following section. The goal is to study the importance of the geomagnetic dipole tilt angle on the plasmasphere-ionosphere properties. After briefly describing the IPIM model, we will present the characteristics of the run conditions, the implied effects on flux tube dynamics, and then the results of the simulation for the inner, intermediate, and outer parts of the plasmasphere. We will finally discuss the results and present a conclusion.

\section{Model}

IPIM is a new model of the ionosphere, first presented in Marchaudon and Blelly (2015). A run on request of planet-independent version of the IPIM model has recently been made available online through the Transplanet website https://transplanet.irap.omp.eu/ hosted by the Plasma Physics Data Centre (CDPP) (Blelly et al., 2019). This model is based on the same philosophy as the high-altitude TRANSCAR model (Blelly et al., 2005). The main differences come from the order moment used to solve the fluid transport equations as well as the magnetic geometry used in the model. TRANSCAR was dedicated to high-altitude studies and used a 13-moment approximation and straight open field lines, while IPIM was initially developed to study inner magnetosphere dynamics, through closed magnetic field lines with dipolar tilted eccentric field lines, but has been recently extended at high-latitudes (Marchaudon et al., 2018). A 16-moment approach has also been implemented in IPIM to allow development of strong temperature anisotropy at high altitude and to accurately model the transition between collision dominated and collisionless media. The main feature of the IPIM model is then the strong variability occurring along a magnetic field line of the solar illumination and of the main forces (gravity and inertia) allowing large diurnal and seasonal variability.

Three main modules form IPIM: a fluid model for six main ions $\left(\mathrm{O}^{+}, \mathrm{H}^{+}, \mathrm{N}^{+}, \mathrm{NO}^{+}, \mathrm{O}_{2}^{+}, \mathrm{N}_{2}^{+}\right)$and thermal electrons; a kinetic model for suprathermal electrons; and a chemical module to take into account all the chemical reactions involving the main charged species formed in the ionosphere. The main characteristic of the IPIM model lies in the coupling between the fluid and kinetic models. The fluid model solves the 16-moment order of the transport equations for the three atomic ions and the thermal electrons whose dynamics will be especially important above the ionospheric F peak region. For the three molecular ions, density is solved independently, while same velocity, temperature, and heat flux are assumed for these heavier species confined at relatively low altitudes and dominated by chemical processes. Finally 


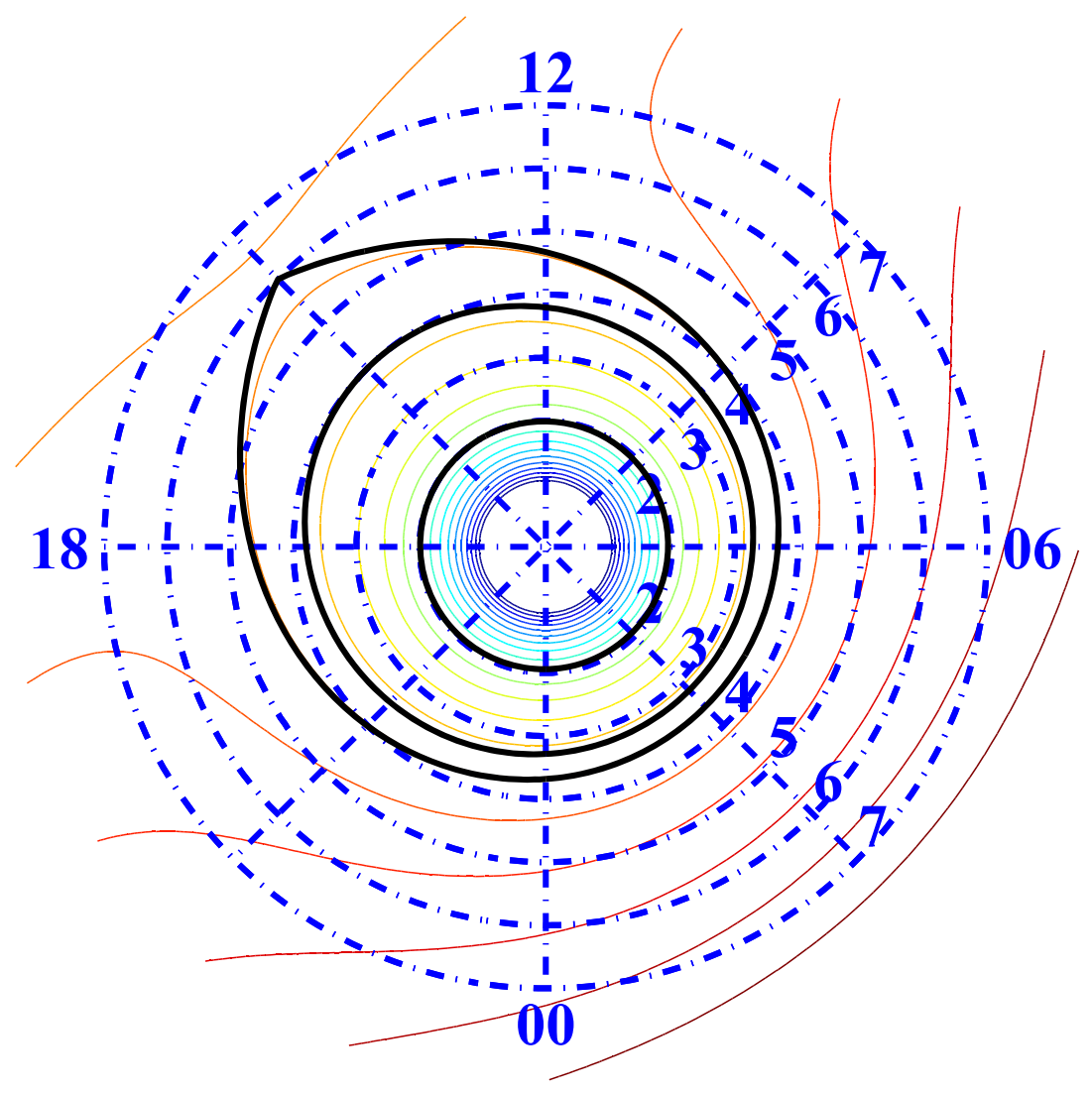

Figure 1. Convection and corotation potential contours in the equatorial plane with a stagnation point at $L_{\max }=6 R_{E}$ and 15 MLT. The equipotential contours followed by the three flux tubes at $L_{\max }=2,4,6 R_{E}$ are represented by black solid lines.

in the near future, we plan to add $\mathrm{He}^{+}$, whose dynamics can be important especially in the transition region between ionosphere and plasmasphere (1,000-10,000 km). With 16-moment approximation, parallel and perpendicular energies (temperature) have their own heat flux. This full separation between parallel and perpendicular is essential for stability as demonstrated by Blelly and Schunk (1993) for electrons. The kinetic model solves the steady state Boltzmann transport equation of the suprathermal electrons along the magnetic field line, taking into account sources (photoionization from primary as well as secondary electrons and precipitation) and losses through collisions with atmosphere and thermal electrons. De facto, this model intrinsically accounts for interhemispheric transport of suprathermals and thus allows for production and heating by electrons coming from the opposite hemisphere. Such processes may be responsible for production associated with sunrise in the opposite hemisphere and are important in the frame of ionosphere/plasmasphere dynamics, but we do not address such effects in this study. The two models are intrinsically linked since the fluid model needs the ionization rates and heating of thermal electrons from the suprathermal electrons given by the kinetic model, and the fluid model gives in return the density and temperature of thermal electrons to the kinetic model.

IPIM uses several inputs, such as the solar flux reconstructed through the Flare Irradiance Spectral Model (FISM, Chamberlin et al., 2007) and the neutral atmosphere parameters (density, temperature) coming from NRLMSISE-00 (Hedin, 1987, 1991; Picone et al., 2002) and horizontal neutral wind from HWM14 model (Drob et al., 2008; Drob et al., 2015; Emmert et al., 2008). Finally, realistic electrodynamics inputs can also be used (convection, precipitation, and field-aligned currents), which are of great use for modeling high-latitudes processes (Marchaudon et al., 2018) but are less important for modeling quiet midlatitudes conditions. Plasma transport equations are solved along magnetic field lines in the IPIM model, implying an Eulerian 1-D description. But the field lines can also be transported in the transverse direction through corotation and convection, implying a Lagrangian 2-D description. This transverse motion may in particular modify the distance of the field line apex with respect to the Earth's center, implying contraction or 
expansion of each element of volume along the field line. This volume variation is taken into account as a specific divergence term in the equations solving intensive quantities such as density. A complete description of the methodology can be found in Marchaudon and Blelly (2015) (see in particular sections 2.1.2 and 2.1.4, figure 1 and the divergence term being described in equation 20).

The competition between corotation and convection modifies the path of external closed flux tubes whose distance from Earth's center will change during a full rotation around the Earth and will imply a variation of the flux tube volume with respect to time. In the present study, a simple analytical model for corotation and convection is used (see next section). In the following, a specific flux tube will be referred by its $L$-shell position (McIllwain parameter) at 15 MLT in the stagnation region and will be called $L_{\text {max }}$, corresponding to the highest $L$-shell value in $R_{E}$ units that the tube will reach during its motion.

\section{Run Conditions}

The simulation runs presented in this paper consist of two different sets of plasmaspheric simulations lasting 30 days each, one ending at the equinox day and one ending at the solstice day (spring and summer in northern hemisphere, respectively). For each simulation run, several closed flux tubes spanning from 1.2 to $6 R_{E}$ have been investigated. The combined corotation-convection model used in these simulations is a simple static analytical model (Schulz, 2007), whose parametrization allows choice of the location in the equatorial plane of the stagnation point, where corotation and convection are exactly balanced and for which the plasmaspheric streamlines pattern is kept unchanged. In this set of runs, we choose a stagnation point very far from Earth at $6.01 R_{E}$ and 15 MLT and mimicking very quiet conditions. The equatorial potential pattern is presented in Figure 1. Such a choice for a constant and quiet potential pattern is mainly motivated by the need to eliminate all sources of variability except those caused by the variation of the dipole tilt angle. For the same reason, the IPIM solar and magnetic activity inputs have been chosen constant over 30 days and for relatively moderate conditions (global magnetic index $\mathrm{a}_{\mathrm{p}}=4$ and solar radio $10.7 \mathrm{~cm}$ flux $\left.\mathrm{F}_{10.7}=150 \mathrm{sfu}\right)$.

In Figure 1, the paths followed by three different flux tubes (black solid curves) have been superimposed on the potential map. These three tubes have their $L_{\max }$ at 2, 4, and $6 R_{E}$, respectively. The closest tube from Earth $\left(L_{\max }=2 R_{E}\right)$ is characterized by an almost circular motion almost fully dominated by corotation. For the tube with $L_{\max }$ at $4 R_{E}$, convection starts to have a perceptible effect, and the trajectory displays a slightly elliptical pattern. Finally, the tube furthest from Earth $\left(L_{\max }=6 R_{E}\right)$ almost attains the stagnation point and thus remains a very long time in this region, resulting in the very angular shape of the trajectory around 15 MLT. This flux tube undergoes a strong expansion before the stagnation point and a strong contraction after. The combined corotation-convection effects on magnetic flux tube dynamics and on the electron density distribution along the different flux tubes are presented in the next sections.

\section{Combination of Motion}

The motion of a flux tube around the Earth is a combination of corotation and convection. As discussed in Marchaudon and Blelly (2015), the tube moves faster than corotation in the 21-09 MLT sector because convection and corotation act the same way, while the tube is slower than corotation in the 09-21 MLT sector, because both circulations are opposite. The differential motion is maximum in the stagnation region around 15 MLT and can force a flux tube with an apex reaching $L_{\max }=6 R_{E}$ to remain more than one day in this sector. This is the reason for the stretching of the equipotential lines in the 15 MLT sector, which is visible in Figure 1 for tubes with an apex at 15 MLT above $L_{\max }=3.5 R_{E}$. However, the effect is not symmetrical, and there is an overall drift of the tube, which makes the tube move slower than corotation. As a matter of fact, in our convection model, a tube corresponding to $L_{\max }=2 R_{E}$ is slower by $0.15 \%$ than corotation, while a tube with $L_{\max }=4 R_{E}$ is slower by $6.4 \%$ and a tube with $L_{\max }=6 R_{E}$ by $58 \%$.

This drift can be highlighted by looking at the variation of the dipole tilt angle projection for a specific tube. The top panel of Figure 2 shows on the colored curve, the temporal variation over 30 days of the tilt angle between the magnetic equatorial plane and the geographic equatorial plane for a flux tube corresponding to $L_{\text {max }}=4 R_{E}$. The superimposed colors correspond to the value of the solar zenith angle $\chi$ at the apex of the tube over time (see the colorbar for the scale). We clearly see the diurnal variations of $\chi$ and of the tilt angle due to the nonuniform rotation of the tube around the Earth. The black curve is the daily evolution of the tilt angle for this flux tube when its apex is located at noon local time. We see that we have filtered 


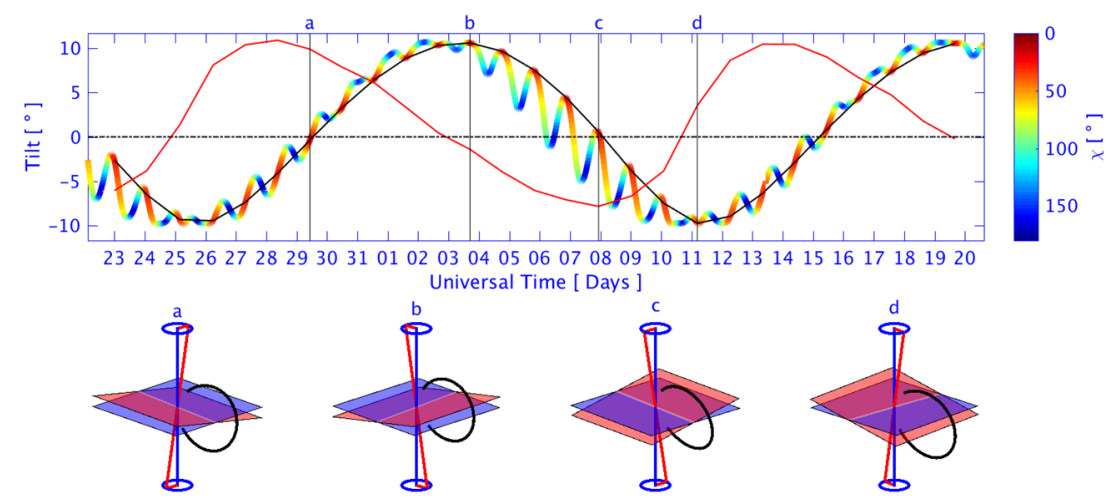

Figure 2. Temporal evolution of the tilt between the magnetic equatorial plane (plane colored in blue in sketches (a), (b), (c), and (d)) and the geographic equatorial plane (plane colored in red in sketches (a), (b), (c), and (d)). The colored curve in the top panel shows the value of the solar zenith angle $\chi$ at the apex of the flux tube with $L_{\max }=4 R_{E}$ (the colorbar gives the scale). The black curve corresponds to the tilt of the tube when its apex is at noon local. The red curve corresponds to the difference angle per day between the motion of this flux tube and the rotating planet. Position (a) corresponds to a tilt of $0^{\circ}$, while the tube is moving faster than the rotating planet, (b) corresponds to the maximum of tilt angle, (c) corresponds to a position with a $0^{\circ}$ tilt angle, while the tube is moving slower than the rotating planet, and (d) corresponds to the minimum tilt angle.

out the diurnal variation and that a long term evolution remains, which corresponds to the drift mentioned before. Due to the diurnal drift of about $6 \%$, more than 15 days are required $\left(15 \simeq \frac{1}{0.064}\right)$ to re-phase the flux tube, meaning coming back to the exact same position with respect to the Earth's surface below. The red curve shows the drift angle's daily rate for the tube with apex at noon local time. This curve shows that when this drift angle velocity is maximum, the variation of the dipole tilt angle projection (diurnal variation of the curve and of its colors) is also maximum. On the bottom row of this figure, the flux tube position is also displayed at different instants labeled (a), (b), (c), and (d) on the top panel, which shows the respective tilt angle between the geomagnetic and the geographic equatorial planes (red and blue respectively) with respect to this flux tube position. Instants (a) and (c) correspond to a $0^{\circ}$ tilt between the two planes while instants $(b)$ and $(\mathrm{d})$ correspond to a maximum tilt $\left(\approx 11.5^{\circ}\right)$ between the two planes. We now describe in detail the motion of the flux tubes depending on their $L_{\max }$ value and the effect it causes on the electron density distribution along the flux tube.

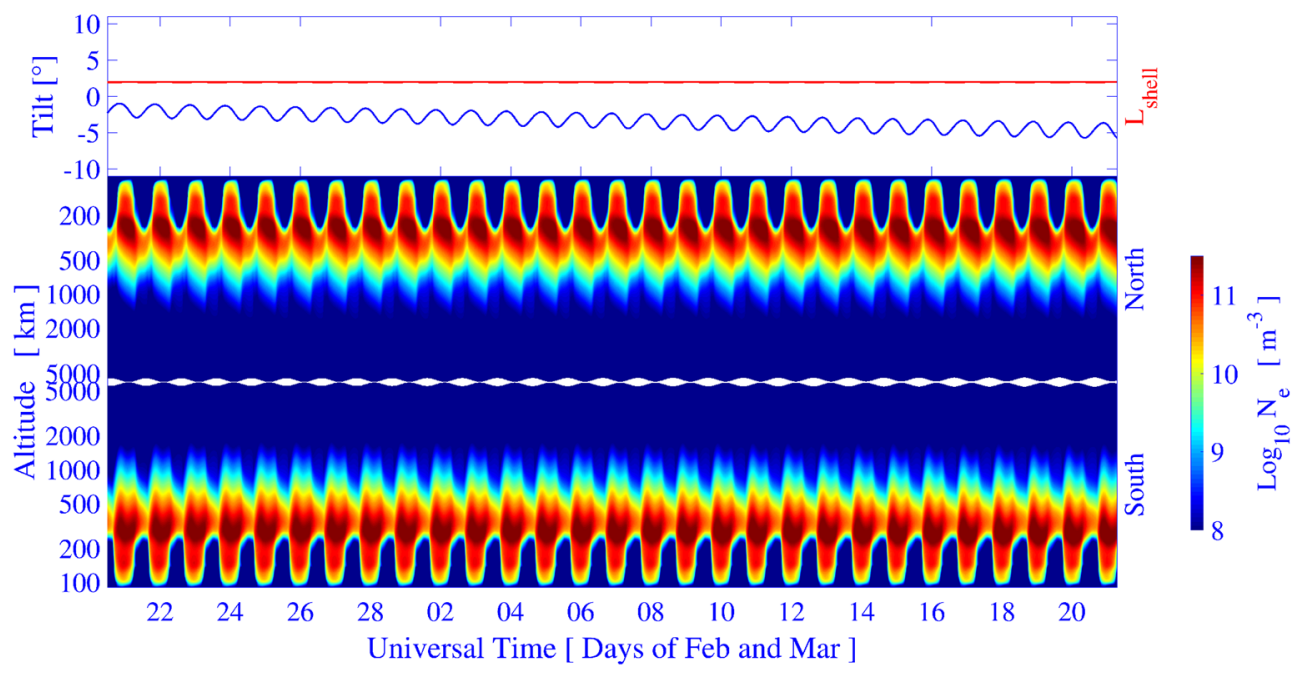

Figure 3. Time/altitude color plot of thermal electron density (bottom panel) along closed field line for $L_{\max }=2 R_{E}$. A period of one month before North spring equinox spanning February and March is displayed as a function of the day of the month. The two hemispheres are specified by labels "South" and "North." In the top panel, tilt angle projection is plotted in blue together with the $L$-shell extension in red, for the same period of time. 


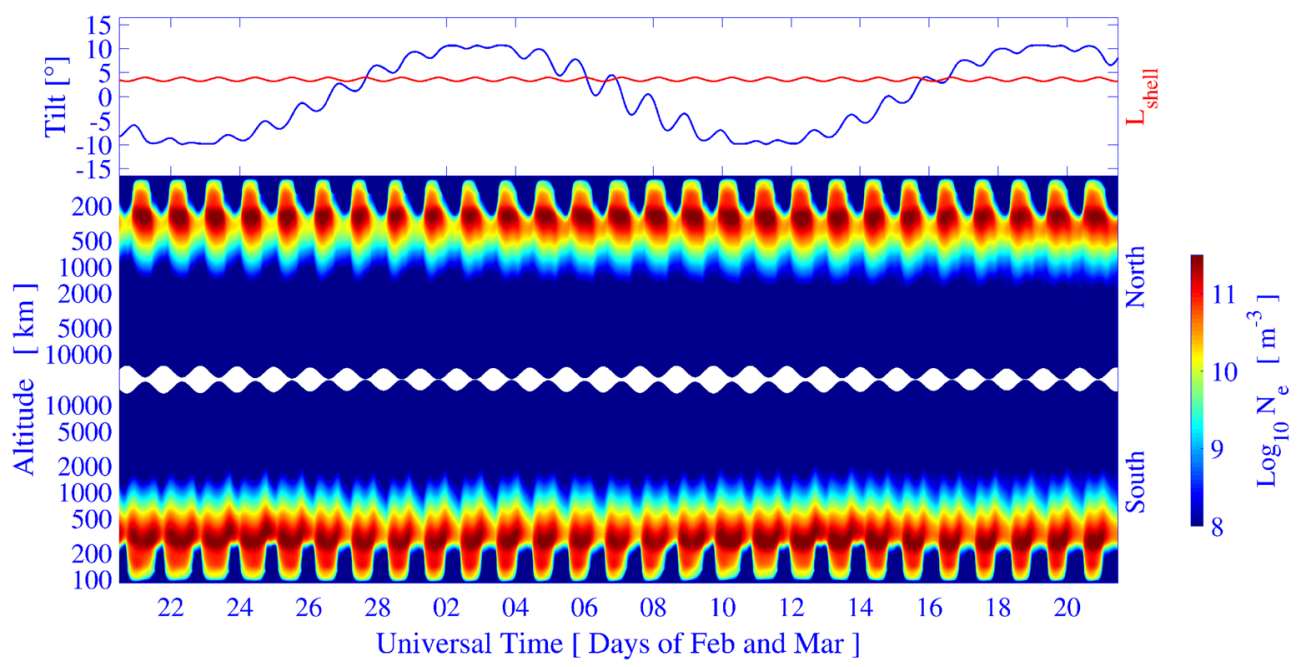

Figure 4. Time/altitude color plot of thermal electron density (bottom panel) along closed field line for $L_{\max }=4 R_{E}$. A period of one month before North spring equinox spanning February and March is displayed as a function of the day of the month. The two hemispheres are specified by labels "South" and "North." In the top panel, tilt angle projection is plotted in blue together with the $L$-shell extension in red, for the same period of time.

\section{Results}

We have looked through the complete 30 days of simulation for the flux tubes with $L_{\max }$ at 2, 4, and $6 R_{E}$ at equinox and with $L_{\max }$ at $4 R_{E}$ at solstice (Figures 3 to 6). For each tube at equinox, the results are presented in the same figure format. The top panel shows the variation of the $L$-shell along the tube path (red line) against Universal Time (UT) for the 30 days of simulation, together with the variation of the dipole tilt angle projection in the flux tube plane with respect to the geographic equatorial plane (blue line), hereafter denoted dipole tilt projection. The bottom panel shows a color-panel plot of the thermal electron density along the complete flux tube for the 30 days simulation displayed with respect to UT ( $x$-axis) and altitude along the flux tube ( $y$-axis in logarithmic scale). Density is also displayed with a logarithmic scale in order to emphasize the dynamics at all altitudes. For the tube at solstice, we have also added a color-panel plot showing the $\left[\mathrm{N}_{\mathrm{H}^{+}}\right] /\left[\mathrm{N}_{\mathrm{e}^{-}}\right]$ratio (middle panel).

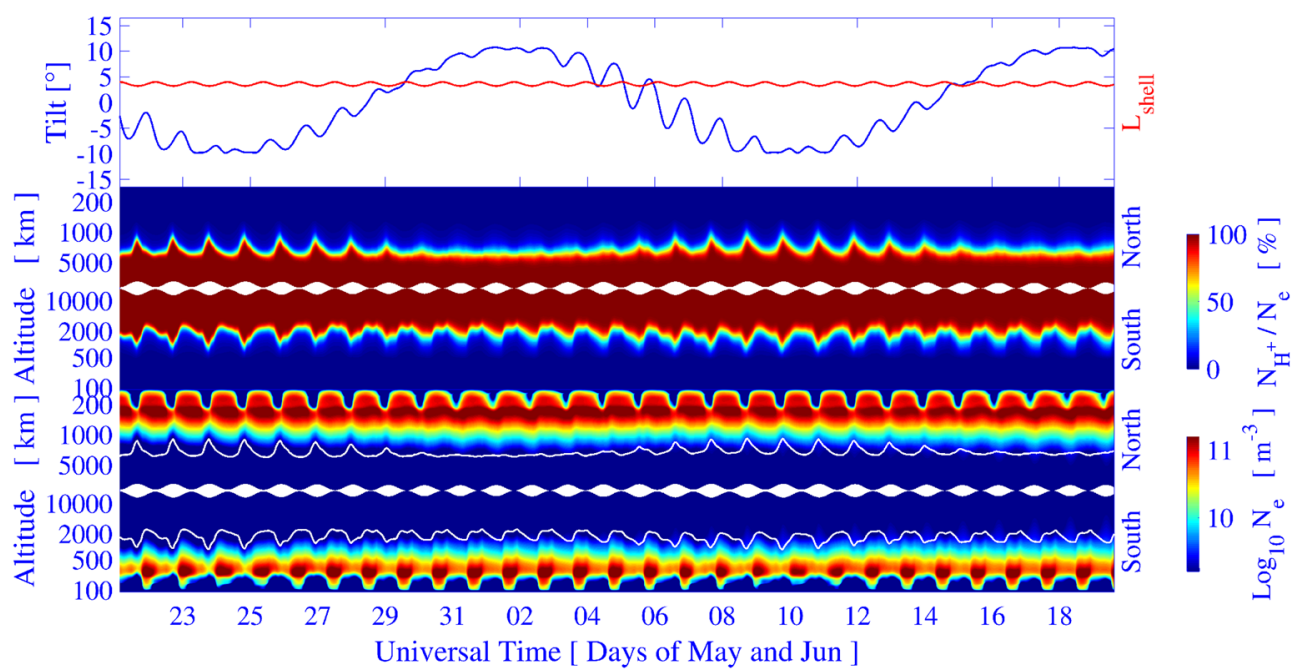

Figure 5. Time/altitude color plots of thermal electron density (bottom panel) and $\left[\mathrm{N}_{\mathrm{H}^{+}}\right] /\left[\mathrm{N}_{\mathrm{e}^{-}}\right]$(middle panel) along closed field line for $L_{\max }=4 R_{E}$. A period of one month before North summer solstice spanning May and June is displayed as a function of the day of the month. The two hemispheres are specified by labels "South" and "North." The white solid curve in the bottom panel indicates $\mathrm{H}^{+} / \mathrm{O}^{+}$upper transition height (denoted $z_{50}$ ) in each hemisphere. In the top panel, tilt angle projection is plotted in blue together with the $L$-shell extension in red, for the same period of time. 


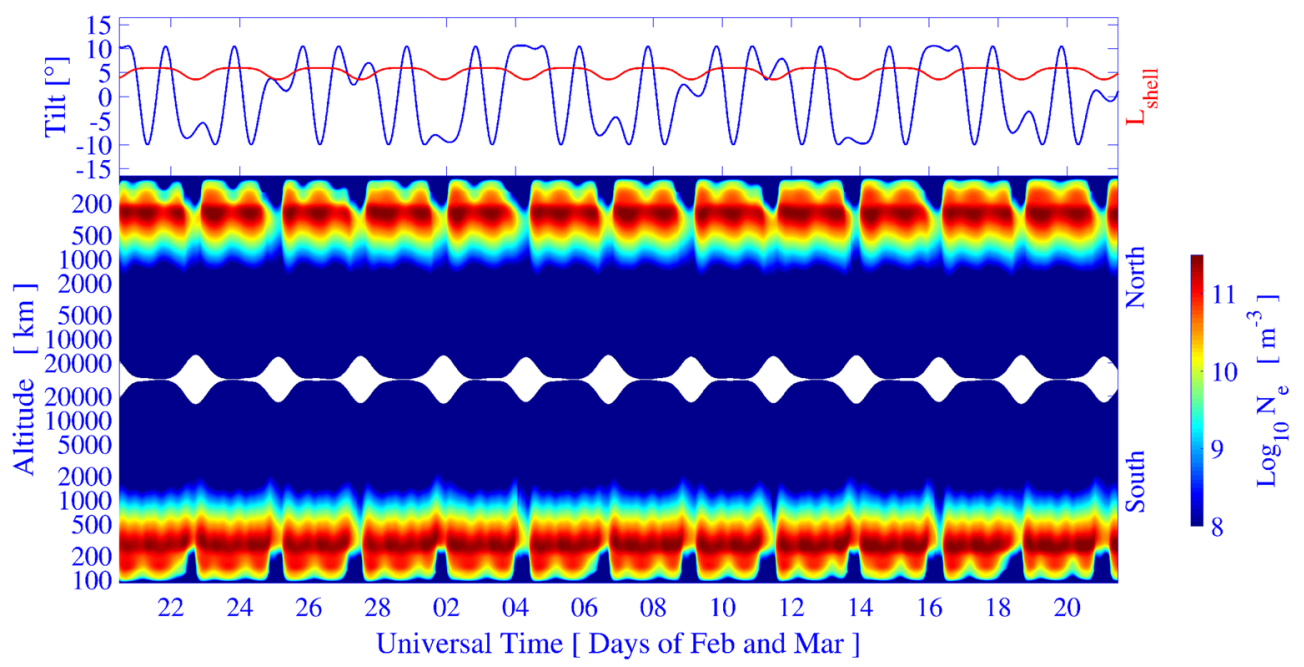

Figure 6. Time/altitude color plot of thermal electron density (bottom panel) along closed field line for $L_{\max }=6 R_{E}$. A period of one month before North spring equinox spanning February and March is displayed as a function of the day of the month. The two hemispheres are specified by labels "South" and "North." In the top panel, tilt angle projection is plotted in blue together with the $L$-shell extension in red, for the same period of time.

\subsection{Inner Plasmasphere}

For the tube at $L_{\max }=2 R_{E}$ at equinox, the results are displayed in Figure 3. Looking at the dipole tilt projection (blue line, top panel), two different motions are observed: a diurnal oscillation of about $2^{\circ}$ in amplitude and a global drift toward more negative angles over the 30 days, from $-2^{\circ}$ to $-5^{\circ}$ in average. These two motions come from the competition between convection and corotation. While minor so close to Earth, the convection effect causes a variation of the distance of the flux tube in the equatorial plane, as seen in the small evolution of the equatorial $L$ distance of the flux tube (red line, top panel) and drives a nonuniform rotation speed of the flux tube around the Earth. The diurnal oscillation is due to the slower speed of the tube before the stagnation region and its faster speed after the stagnation region with respect to the Earth's rotation speed. This rotation speed variability implies that the dipole tilt projection oscillates slightly in the course of a UT day. The convection effect also causes a slower average rotation speed of the flux tube with respect to the Earth's rotation speed. This difference in average rotation speed implies that the tube will take slightly longer than 24 UT hours to complete a full turn around the Earth and causes a drift of the dipole axis with respect to the flux tube plane leading to a slow decrease of the dipole tilt projection.

The oscillation of the dipole tilt projection also causes a small oscillation with time of the solar zenith angle $(\chi)$ on each point of the flux tube. However in the case of the $L_{\max }=2 R_{E}$ tube, this $\chi$ oscillation remains small and is totally outclassed by the usual diurnal variation of $\chi$ caused by the corotation. In consequence, for each hemisphere taken separately, the electron density distribution along the flux tube displays an almost identical day-to-day pattern (bottom panel of Figure 3). On the other hand, the slow drift of the tilt projection may explain the hemispheric differences in the electron density pattern, which does not become identical even at equinox day. Such an identical pattern could probably have been observed for a flux tube only if the dipole tilt projection was close to $0^{\circ}$ at equinox day.

\subsection{Intermediate Plasmasphere}

For the tube at $L_{\max }=4 R_{E}$ at equinox, the results are displayed in Figure 4 . The $L$-shell along the tube path (red curve, top panel) shows an oscillation of stronger amplitude than at $L_{\max }=2 R_{E}$, and the dipole tilt projection displays a more complex signal with two different periods (blue curve, top panel). Each period corresponds again to the two convection effects described in the $L_{\max }=2 R_{E}$ case. The smallest diurnal oscillation is caused by the nonuniform rotation speed of the flux tube with a deceleration before the stagnation point and an acceleration after the stagnation point. However, since this tube is further from Earth, the effect is amplified with stronger deceleration and acceleration leading to the oscillation amplitude of the dipole tilt projection reaching almost $10^{\circ}$ (see blue curve behavior for days 5-6 March). 


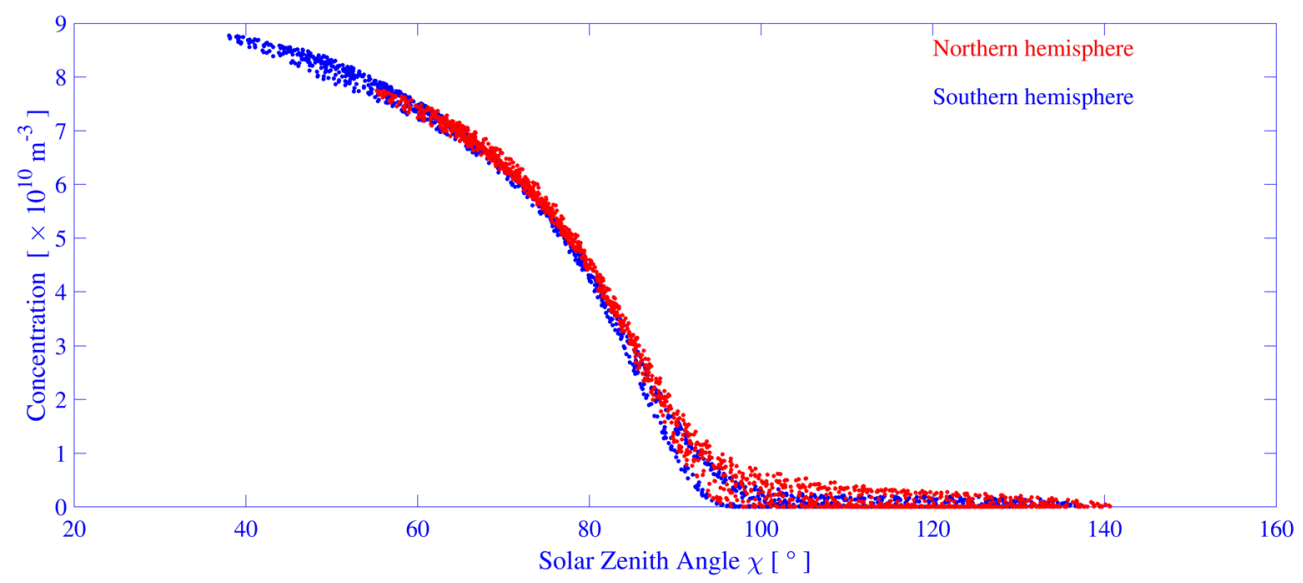

Figure 7. Electron concentration at $180 \mathrm{~km}$ in southern (in blue) and northern (in red) hemispheres for the closed field line with $L_{\max }=4 R_{E}$, as a function of solar zenith angle $\chi$ and for the period of one month before North spring equinox.

The longer period of about 16.5 UT days is due again to the lower average rotation speed of the flux tube with respect to the Earth's speed, causing a drift of the dipole axis with respect to the flux tube plane. However, this average speed is now perceptibly lower than the Earth's speed (by 6.4\% as already stated in section 4), causing the Earth to accomplish a full supplementary rotation with respect to the flux tube in about 16.5 UT days. The minimum at about $-11.5^{\circ}$ and maximum at about $+11.5^{\circ}$ correspond to the two positions where the dipole axis is contained in the flux tube plane (minimum when the North pole is in phase with the flux tube), and the $0^{\circ}$ crossings correspond to the dipole axis perpendicular to the flux tube plane.

Finally, the amplitude of the diurnal oscillations is not constant over time, stronger when the dipole tilt projection passes from $+11.5^{\circ}$ to $-11.5^{\circ}$ (on descending branch) than when it passes from $-11.5^{\circ}$ to $+11.5^{\circ}$ (on ascending branch). This asymmetrical effect could be due to the drift angle velocity being maximum during this ascending branch causing the variation of the dipole tilt projection to also be maximum, as already stated in section 4 and seen with the red curve in Figure 2. These effects lead to strong $\chi$ diurnal oscillations on each point of the flux tube, as well as strong day-to-day $\chi$ variability along the flux tube causing in response large variability of the solar illumination, of the atmosphere ionization and of the resulting electron density distribution (bottom panel of Figure 4). It is even possible to recognize in the electron density pattern the two periods seen in the dipole tilt projection plot (top panel of Figure 4).

In each hemisphere, a clear day-to-day variation is observed in the diurnal pattern of the electron density but with an almost identical pattern being reproduced every 16.5 UT days (see, e.g., days 25 February and $12 \mathrm{March}$ ). Finally, for the same reason as for the tube at $L_{\max }=2 R_{E}$, both hemispheres display clear diurnal asymmetries even at equinox day, although the ionospheric $F$ region and $E$ region average densities are roughly of the same order of magnitude. As for $L_{\max }=2 R_{E}$, we attribute this asymmetry to a nonzero dipole tilt projection at equinox day. To address the question of this asymmetry, we looked at the temporal evolution of the electron density at $180 \mathrm{~km}$. This altitude was chosen because it corresponds more or less to the transition region between molecular ions (mainly $\mathrm{NO}^{+}$) and the atomic ions (mainly $\mathrm{O}^{+}$) and is still strongly dominated by chemical equilibrium between photoproduction and $\mathrm{NO}^{+}-e^{-}$dissociation recombination. Figure 7 presents the electron concentration at this altitude in the southern (in blue) and the northern (in red) hemispheres as a function of solar zenith angle $\chi$, for the period covered by the simulation. We clearly see that both hemispheres have the same variation of the electron density with $\chi$, but the asymmetry comes from the lower $\chi$ extension in the northern hemisphere compared with the southern hemisphere for this pre-spring period. $\chi$ values below $60^{\circ}$ are not reached in the northern hemisphere because first, we are going from winter to spring, and the geographic latitude of the northern footprint is located above $50^{\circ}$ and then, the $\chi$ extension depends on the tilt angle (see Figure 2). The shape of this variation is characteristic of a Chapman layer, and the important point is that the ionosphere disappears just after sunset $\left(\chi \geq 90^{\circ}\right)$. So, we have an unambiguous relationship, at $180 \mathrm{~km}$, between electron concentration and solar zenith angle, which thus provides a marker for the duration of the daylight (nonzero concentration) and darkness (almost zero concentration) periods (see section 6). 
We also present results for the tube $L_{\max }=4 R_{E}$ at solstice in Figure 5. As for $L_{\max }=4 R_{E}$ at equinox, the two periods are also visible in the tilt angle projection (blue line, top panel), as well as in the $\left[\mathrm{N}_{\mathrm{H}^{+}}\right] /\left[\mathrm{N}_{\mathrm{e}^{-}}\right]$ ratio (middle panel) and in the electron density profile (bottom panel). Moreover, amplitude variations of the diurnal oscillation are also observed and are clearly emphasized during solstice with oscillations almost completely smoothed out on the ascending branch and strongly enhanced on the descending branch of the longest period (16.5 UT days). This effect is probably emphasized during solstice by the tilt of the geographic equatorial plane of about $+23.5^{\circ}$ with respect to the normal to the ecliptic plane. As expected, the solstice conditions also produce asymmetric behavior between hemispheres with a very strong and almost continuous ionospheric $\mathrm{F}$ region in the summer northern hemisphere (bottom panel), due to $\chi$ being almost always above $90^{\circ}$ causing continuous ionization. This is particularly evident when the dipole tilt projection is at its maximum value around $+11.5^{\circ}$ (Days 1 to 5 ) corresponding to a dipole inclination being close to $+34^{\circ}$ from the normal to the ecliptic plane, enhancing further the atmosphere's photoionization. These effects also strongly affect the dynamics of the plasma at the interface between the ionosphere and the plasmasphere, which is here roughly estimated as being at a $0.5\left[\mathrm{~N}_{\mathrm{H}^{+}}\right] /\left[\mathrm{N}_{\mathrm{e}^{-}}\right]$ratio (see middle panel but also the white lines on bottom panel). It is then clear on this plot that this interface also varies with altitude, with a diurnal cycle (varying from about 1,000 km during night to 4,000 km during day) but also with the longer period variation in dipole tile projection. It is flatter for a dipole tilt projection around $+11.5^{\circ}$ (e.g., between 30 February and $6 \mathrm{March}$ ) especially in the summer hemisphere and more symmetrical between hemispheres around $-11.5^{\circ}$.

\subsection{Outer Plasmasphere}

Figure 6 displays the results obtained at equinox for a flux tube reaching $L_{\max }=6 R_{E}$, very close to the stagnation point. As expected, the convection effect is strongly enhanced and affects the dynamics of the plasma. Despite not being chaotic, the variation of the dipole tilt projection becomes extremely complex (blue curve, top panel). The $L$-shell along the tube path (red curve, top panel) shows that a full rotation of the flux tube around the Earth is now accomplished in about 48 hours since the average rotation speed of the tube is considerably slower than the Earth's (by 58\% as already stated in section 4). This results in an electron density characterized by a periodic 2-day enhancement of ionization, in phase but asymmetric, in both hemispheres (bottom panel). The temporal structure of the density is clearly an effect of the dipole tilt drift (blue curve, top panel), which makes a full turn with the Earth while the tube remains in the stagnation region (red curve, top panel). This is clearly visible in the dipole tilt projection (blue curve, top panel), for example, on days 25-27 February where the dipole tilt projection spans from $+11.5^{\circ}$ to $-11.5^{\circ}$ and to $+11.5^{\circ}$ again while $L$-shell remains almost constant at about $6 R_{E}$, in the stagnation region. This $\sim 23^{\circ}$ extension has two main effects on the ionosphere. First of all, the solar zenith $\chi$ angle along the flux tube is impacted and evolves with time with an extension controlled by the tilt angle, though the tube remains at the same solar local time. This causes north-south asymmetry which affects the electron density in both hemispheres.

The $\chi$ variation is important at high-latitude and affects ionization production rates. As the $\mathrm{E}$ region is dominated by chemical processes, with time scale of about $1 \mathrm{~min}$, it is very sensitive to this $\chi$ variation, causing the density fluctuation below $200 \mathrm{~km}$ in both hemispheres. Beside this, the F region time scale is about half an hour and is controlled by the balance between production and field aligned transport; thus, it is also very sensitive to the nonuniform motion of the flux tube. Indeed, in a standard ionosphere, the location of the main F peak is controlled by the neutral wind, through its field aligned projection. The rate of variation of the tilt angle (mainly the sign of the variation) affects this field aligned projection and can displace sensitively the location of this peak. This effect is asymmetric between $+11.5^{\circ}$ to $-11.5^{\circ}$ and $-11.5^{\circ}$ to $+11.5^{\circ}$ variations and results in a highly variable ionosphere in the $\mathrm{F}$ peak region. This explains the sawtooth oscillations observed above $200 \mathrm{~km}$ altitude in the ionospheric day-like electron density in both hemispheres (bottom panel). If the density variations below the $\mathrm{F}$ peak follow more or less the variations of the tilt angle (and thus $\chi$ angle), the variations above this peak are more complex due to this balance between production and diffusion. Seasonal effects on the atmosphere (especially the wind) explains the asymmetrical behavior above the F peak between northern and southern hemispheres, even close to equinox with similar illumination in both hemispheres.

Finally, for the longer periodic variation, a quasi-period of about 12 days can be identified, but the observed pattern is not exactly identical. It corresponds to the time duration needed for the dipole tilt projection to come almost exactly back to the same configuration with respect to the flux tube plane. 


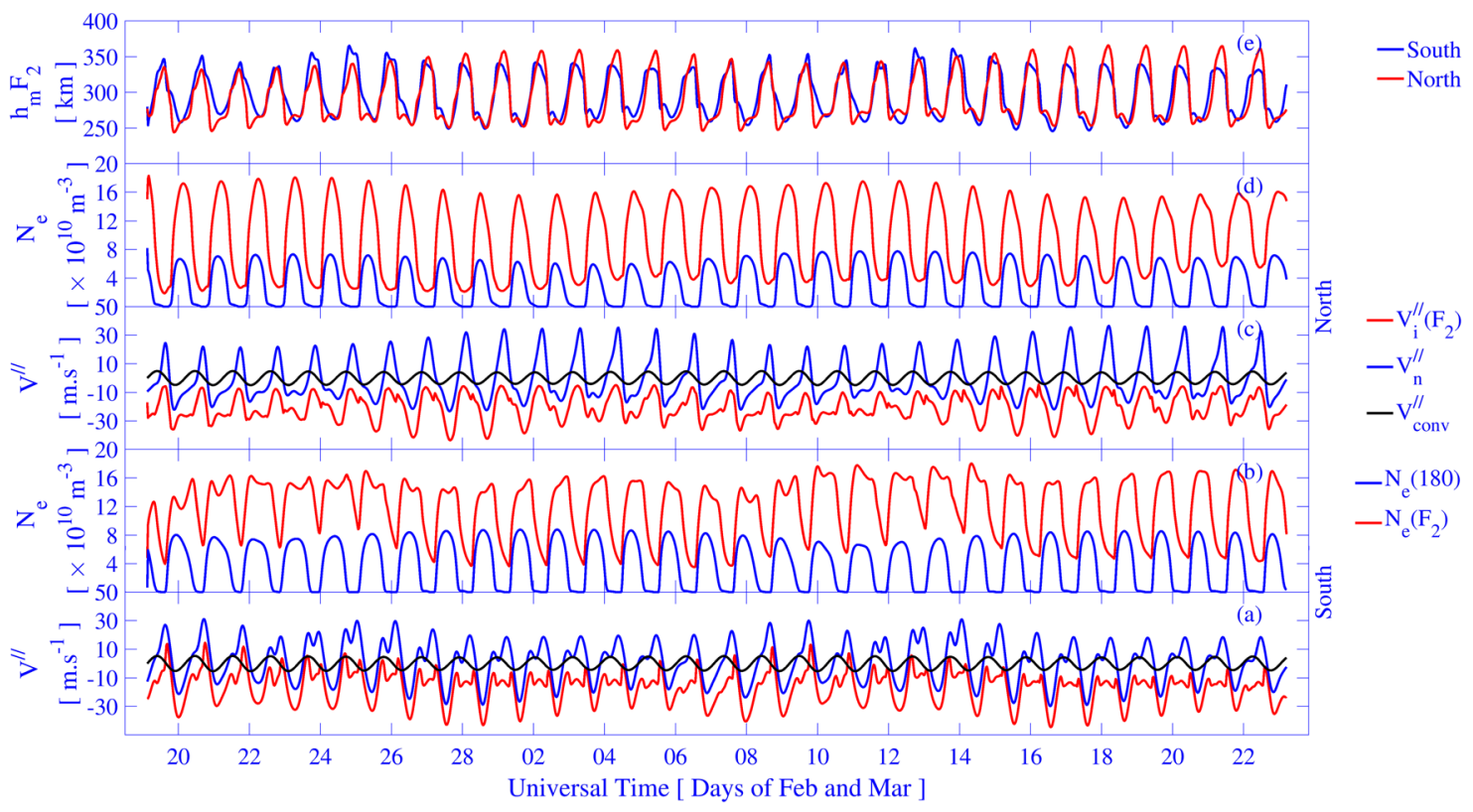

Figure 8. Temporal evolution of the field-aligned ion velocity at $F_{2}$ peak (in red) and neutral velocity at $180 \mathrm{~km}$ (in blue) in panels (a) and (c), with the convention that positive velocity corresponds to upward motion, the electron density at $180 \mathrm{~km}$ (in blue) and at the $F_{2}$ peak (in red) in panels (b) and (d) and $h_{m} F_{2}$ altitude in southern (in blue) and northern (in red) hemispheres in panel (e), for the closed field line with $L_{\max }=4 R_{E}$. A period of one month before North spring equinox spanning February and March is displayed as a function of the day of the month. Panels (a) and (b) correspond to the southern hemisphere and panels (c) and (d) to the northern hemisphere. The field aligned contribution of the convection is displayed in black on velocity plots (panels (a) and (c)).

\section{Field-Aligned Contributions of Neutral Wind and Convection}

Figures 3 to 6 highlight the complex behavior of the $F$ region, which significantly differs in the southern and northern hemispheres and are most probably related to dynamics of the neutral wind and the convection.

To illustrate this effect, which is responsible for North-South asymmetry, we focus on the simulation for spring equinox with $L_{\max }=4 R_{E}$ (Figure 4) and more specifically on the variations of the $F_{2}$ peak density. Indeed, the density at the peak altitude, hereafter designated as $h_{m} F_{2}$, results from the balance between photoproduction and field-aligned transport and thus characterizes well transport effects. Moreover, $h_{m} F_{2}$ is located just above the region where neutral atmosphere strongly controls the ionospheric dynamics that can be symbolized by the $180 \mathrm{~km}$ altitude that we already used in section 5.2.

On Figure 8, we plot the temporal evolution of the field-aligned ion velocity at the $F_{2}$ peak (in red) and of the neutral velocity at $180 \mathrm{~km}$ (in blue) in panels (a) and (c), with the convention that positive velocity corresponds to upward motion, the electron density at $180 \mathrm{~km}$ (in blue) and at the $F_{2}$ peak (in red) in panels (b) and (d) and the $h_{m} F_{2}$ altitude in southern (in blue) and northern (in red) hemispheres in panel (e). The period of one month before North spring equinox spanning February and March is displayed as a function of the day of the month. Panels (a) and (b) correspond to the southern hemisphere and panels (c) and (d) to the northern hemisphere. The field aligned contribution of the convection is also displayed in black on velocity plots (panels (a) and (c)). Density at $180 \mathrm{~km}$ is used as a marker of daylight and darkness periods for which we clearly see the typical Chapman layer diurnal evolution, as stated in section 5.2. If we look at the $F_{2}$ peak density in both hemispheres, we can notice a strong density enhancement at sunrise followed by a strong density decrease well after sunset, during darkness period. However, northern hemisphere shows a sharp maximum while in southern hemisphere density shows some kind of high value plateau, which lasts longer than in the opposite hemisphere with some kind of temporal oscillations. These oscillations exactly correspond to what is observed on Figures 3 to 6.

Panels (a), (c), and (e) show that the $F_{2}$ region is controlled by neutral wind. Indeed, there is a clear correlation between the dynamics of the wind and $h_{m} F_{2}$, the altitude of the $F_{2}$ peak. As a matter of fact, $h_{m} F_{2}$ has an almost linear dependency on the neutral wind, whatever the hemisphere, with an altitude increasing 
from 240 to $370 \mathrm{~km}$ when the wind increases from -30 to $+40 \mathrm{~m} \cdot \mathrm{s}^{-1}$. This component of the wind is the projection of the horizontal neutral wind along the magnetic field line and so this field-aligned component is a mixture of meridional and zonal components, though the meridional component dominates. Consequently, we can affirm that the $F_{2}$ region is controlled by meridional wind in the lower $F$ region, where collisions on neutrals are strong enough to ensure that the field-aligned ion velocity is very close to the neutral one.

The interesting point is that, in both hemispheres during the darkness period just before sunrise, the neutral wind is turning positive (panels (a) and (c)), imposing $h_{m} F_{2}$ to increase. The ionospheric plasma is then uplifted by the motion of the lower $F$ region, but as the ion velocities at $h_{m} F_{2}$ remain negative, the $F_{2}$ region is broadened in altitude, with no production as we still are in darkness. The result is a distribution of the plasma on a larger altitude range, which leads to a sharp decrease of the $F_{2}$ peak density that is observed before sunrise; transport is then a loss term for $F_{2}$ plasma.

There is no doubt concerning the strong increase at the $F_{2}$ peak: sunrise produces ions and electrons that can only be removed by transport (see panels (b) and (c)). It occurs that the sunrise coincides with a neutral wind turning negative in both hemispheres, resulting in a decrease of $h_{m} F_{2}$. The plasma in the $F_{2}$ region is then downlifted to the $F_{1}$ region while the production increases. As the characteristic time in the $F_{2}$ region is larger than half an hour, there is some delay before transport can balance the production, but overall, the density increases during the ascending phase of the Sun (i.e., $\chi$ is decreasing) and reaches a maximum after zenith, in the descending phase.

However, the neutral velocity differs in the two hemispheres, leading to a different behavior of the $F_{2}$ region. As a matter of fact, neutral wind in the northern hemisphere shows a sharp negative foray and then an almost constant velocity for the rest of the daylight period (panel (d)). This results in a rapid decrease of $h_{m} F_{2}$ to the lowest value at sunrise. The balance between production and transport then leads to a single sharp maximum for the density. On the other hand, the wind in the southern hemisphere turns negative during a longer period of time, which lasts the daylight period. The consequence is that $h_{m} F_{2}$ decreases more smoothly to the minimum value, which is reached approximately when Sun is at zenith, with a strong impact on production/transport balance in the $F_{2}$ region. The density shows a local decrease (panel (b)) when $h_{m} F_{2}$ reaches its minimum because production starts to decrease while the ion velocity reaches its most negative value: $F_{2}$ plasma is removed faster. This explains the apparent oscillation of the plasma in the $F_{2}$ region.

Beside this, the motion of the flux tube imposes an uplifting or downlifting of the plasma and of the altitude grid associated with the tube (in lagrangian representation): the rate of change of this altitude grid with respect to the ground is the field aligned contribution. Its contribution does not appear directly in the velocity but instead, on the advection term present in the transport equations. Even a low, but long lasting, value of this contribution, as it occurs close to the stagnation region, may have a cumulative effect on the ionospheric density. In this simulation, this contribution is oscillating between -5 and $+5 \mathrm{~m} . \mathrm{s}^{-1}$ (because of a moderate convection velocity) and is then not negligible compared to neutral wind. As it is alternately in phase during darkness (before sunrise) and antiphase during daylight (before sunset) with neutral velocity, it contributes mainly to amplify the decrease of electron density in the $F_{2}$ region during darkness, before sunrise.

We can affirm that the $F_{2}$ region is very sensitive to the orientation of the neutral wind, mainly the meridional wind, with respect to the convecting field line at the two footprints. We are in the period before spring coming from winter solstice, and as Drob et al. (2015) showed in their Figure 3, northward wind behaves differently at high latitudes around $50^{\circ}$ (where are located the footprints). In both hemispheres, a sharp transition is observed before sunrise and after sunset with a low minimum reached around noon in the southern hemisphere but a strong maximum reached in the morning sector in the northern hemisphere. This effect is likely to be amplified with the $L$-shell as the convection affects the temporal evolution of the local time along the magnetic field line, which is the driver for the wind. This highlights the importance of the convection, which is responsible for geometrical effects that may have local impact as the one discussed here but also a global impact.

\section{Discussion}

As seen in the previous sections, the simple modeling of closed plasmaspheric flux tubes for stable and quiet conditions shows unexpected complex temporal variations of the plasma distribution along the flux tubes, especially in the ionosphere in both hemispheres. These variations are caused by the competition between 
corotation and convection, which impacts the motion of plasmaspheric flux tubes and causes a subcorotation of the plasmasphere associated with an angular variation of the magnetic dipole tilt projection in the flux tube plane.

The induced differential rotation between the Earth and the flux tube changes the solar illumination along the flux tube and thus the ionospheric plasma production and causes a day-to-day variation in the dynamics of the plasmasphere-ionosphere system. This effect is emphasized by the tilt angle variation rate, which affects the field-aligned transport, mainly through the projection of the horizontal wind along the field line, as discussed in the previous section. These effects increase strongly with the distance of the flux tube from the Earth $\left(L_{\max } \geq 3 R_{E}\right)$, although already discernable at $L_{\max }=2 R_{E}$ and produce ionosphere asymmetry in both hemispheres even close to equinox day, if in particular the dipole tilt projection is far from $0^{\circ}$, as already stated in sections 5.1 and 5.2.

When searching for observations of such an effect, we found some references of conjugate observations at midlatitudes coming from ionosondes (Kalita et al., 2016; Timoçin et al., 2018), GNSS Total Electron Content (TEC) (Gulyaeva et al., 2013), or Radio-Occultation satellite data (Liu et al., 2011). In these papers, average ionospheric density magnitude (given by the local F2 peak region or by TEC) is relatively similar in both hemispheres but displays some asymmetric behavior at specific local time. Although these results could support our finding, when discussed, they are often related to other effects, such as the fixed offset between geomagnetic and geographic coordinates system, which in particular can change the underlying thermosphere properties. This effect is real, and the dipole tilt projection drift could simply add to this already existing complexity.

In the inner plasmasphere, the equilibrium will be even more perturbed by the thermospheric properties (wind and/or subcorotation) thought to be responsible for the ionospheric dynamo disturbance but which are not taken into account in these simulations.

In the outer plasmasphere, magnetic mirror force is an important contribution to the plasma field aligned motion, which is highlighted by the guiding center approximation. However, in the fluid approach, accounting for this effect is only possible if anisotropy is described by the transport equations, as this mirror force is intrinsically associated with energy distribution between parallel and perpendicular component. Marchaudon and Blelly (2015) discuss the contribution of the mirror force in the frame of IPIM and showed how significant its contribution can be (see in particular their figure 8 and associated discussion). As the variable drift associated with the tilt will strongly affect the plasma distribution, giving rise to strong electron and ion anisotropies, or equivalently, to magnetic mirror forcing, the 16-moment approximation used in IPIM is the appropriate approach to catch the effects of the transport in a nonuniform magnetic field. However, as this effect is not central for this paper, we will not discuss it any further.

Although run for very simplified conditions, these simulation results tend to show that no real dynamical equilibrium can be reached in the plasmasphere-ionosphere system, even without an external driver during quiet and stable conditions.

These results seem also to imply that the classical UT-MLT representation of the plasmasphere-ionosphere system is largely inadequate and that it is not possible to have a representative instantaneous snapshot of the plasmasphere by taking several flux tubes spanning different distances from Earth and making them simply rotate a full turn around the Earth. As all these flux tubes will not rotate at the same speed, they will get different dipole tilt projections at a given MLT resulting in an irregular pattern of the plasmaspheric plasma. To get a reliable snapshot of the plasmasphere at a given time, it is thus necessary either to use a model with a full Eulerian 3-D description of the plasmasphere-ionosphere system or to consider in IPIM simultaneously a large number of flux tubes evenly distributed throughout the plasmasphere. Even then, the flux tube distribution will soon become uneven, especially at large distance from Earth $\left(L_{\max } \geq 3 R_{E}\right)$, due to their nonuniform rotation around the Earth, with more tubes concentrated just before the stagnation region and largely less tubes elsewhere, especially after the stagnation region.

\section{Conclusion}

The simulations presented in this paper were an attempt to deepen our understanding of the dynamics of the plasmasphere-ionosphere system and to give a new view on the capacity for this system to reach equilibrium or not. To do so, we ran the IPIM model for several flux tubes at different distances from Earth for 
long periods (30 days) close to solstice and equinox and for very quiet conditions. As expected, the plasma distribution along each flux tube shows the strong influence of the variable solar illumination, but more unexpectedly, the rate of variation of the dipole tilt projection, associated with the nonuniform rotation of the flux tube, enhances this influence and impacts the field-aligned transport. This significantly affects the plasma distribution of the flux tube and emphasizes the asymmetrical distribution between the two hemispheres as well as the temporal evolution of the F region. This nonuniform motion is due to the competition between corotation and convection, which causes eventually a lag of the flux tube motion with respect to the Earth. This combination of nonuniform rotation and subcorotation in average becomes important especially for $L_{\max } \geq 3 R_{E}$ and causes nonuniform drift of the dipole tilt projection in the plane of the flux tube with time, changing drastically the plasma distribution along the tube but also from one tube to the other depending on its initial position with respect to the dipole tilt orientation at the beginning of the simulation. Although subcorotation is already a well-known process in the plasmasphere, the implication it can have on the plasma distribution along a plasmaspheric flux tube has not yet been studied to our knowledge. This effect is probably weaker than thermospheric (e.g., variable winds) or external (e.g., variable convection) factors, but these simulation results seem to show that no dynamical equilibrium can be reached along a plasmaspheric flux tube even for extremely quiet and stable conditions for $L_{\max } \geq 3 R_{E}$. These results should be compared to simulation runs, where $\mathrm{He}^{+}$is taken into account in IPIM to confirm the importance of the $\mathrm{He}^{+}$ions in the field-aligned dynamics of the ionosphere-plasmasphere system. Finally, these results should also be incorporated to other simulations taking into account variable thermospheric winds or magnetospheric convection, in order to unveil the importance of the contribution of each effect.

These simulation results give insight into the global dynamics of the plasmasphere-ionosphere system and prove somehow that it is illusory to look for an equilibrium state of this system even during equinox and even for very stable and quiet solar wind and magnetospheric conditions. This also has strong implications on the signal variability, which could be expected between different ground-based stations (ionosondes, Incoherent Scatter Radar, ground-based magnetometers) as well as satellites. Since one ground-based station is at a fixed geographic location, we cannot directly capture the effect of the drift of the tilt angle projection. To do so, we would need to follow with a specific space mission the same field line for a certain time; this is impossible, since it implies to have a satellite drifting in the system with a velocity equivalent to the combined convection and corotation drift. However, in case of ground-based stations, even for stations located at almost identical magnetic latitudes but separated in longitudes, the variations implied by this different dipole tilt projection could significantly change physical quantities from one station to the other. Moreover, even different satellites crossing the same MLT of the plasmasphere at different universal times may give rise to non-negligible variations of the dipole tilt projection and may make comparisons difficult. Finally, concerning GNSS satellites, the various lines-of-sight with several ground based stations will encompass a large number of flux tubes with different motion histories. This will affect the TEC and impact ionospheric reconstruction, especially in the outer plasmasphere, where the variations in the F region discussed before are superimposed to the density fluctuations of the subauroral region.

Acknowledgments

An online version of IPIM is available on transplanet web interface (https:// transplanet.irap.omp.eu/) and the simulations made for this study are available at the following link https:// transplanet.irap.omp.eu/published. $\mathrm{html}$. IPIM model is a property of CNRS. This work is supported by the Programme National Soleil Terre (PNST) from Institut des Sciences de l'Univers of Centre National de la Recherche Scientifique (INSU/CNRS) co-funded by CNES and CEA. This work was granted access to the HPC resources of CALMIP supercomputing center under the allocation 2015-P1520.

\section{References}

Blelly, P. L., Lathuillère, C., Emery, B., Lilensten, J., Fontanari, J., \& Alcaydé, D. (2005). An extended TRANSCAR model including ionospheric convection: Simulation of EISCAT observations using inputs from AMIE. Annales Geophysicae, 23, 419-431. https://doi.org/10. 5194/angeo-23-419-2005

Blelly, P. L., Marchaudon, A., Indurain, M., Witasse, O., Amaya, J., Chide, B., \& Bouchemit, M. (2019). Transplanet: A web service dedicated to modeling of planetary ionospheres. Planetary Space Science, 169, 35-44. https://doi.org/10.1016/j.pss.2019.02.008

Blelly, P. L., \& Schunk, R. W. (1993). A comparative study of the time-dependent standard 8-, 13- and 16-moment transport formulations of the polar wind. Annales Geophysicae, 11, 443-469.

Brice, N. M. (1967). Bulk motion of the magnetosphere. Journal of Geophysical Research, 72, 5193-5211. https://doi.org/10.1029/ JZ072i021p05193

Burch, J. L., Goldstein, J., \& Sandel, B. R. (2004). Cause of plasmasphere corotation lag. Geophysical Research Letter, 31, L05802. https:// doi.org/10.1029/2003GL019164

Chamberlin, P. C., Woods, T. N., \& Eparvier, F. G. (2007). Flare Irradiance Spectral Model (FISM): Daily component algorithms and results. Space Weather, 5, 7005. https://doi.org/10.1029/2007SW000316

Clilverd, M. A., Meredith, N. P., Horne, R. B., Glauert, S. A., Anderson, R. R., Thomson, N. R., \& Sandel, B. R. (2007). Longitudinal and seasonal variations in plasmaspheric electron density: Implications for electron precipitation. Journal of Geophysical Research, 112 , A11210. https://doi.org/10.1029/2007JA012416

Clilverd, M. A., Smith, A. J., \& Thomson, N. R. (1991). The annual variation in quiet time plasmaspheric electron density, determined from whistler mode group delays. Planetary Space Science, 39, 1059-1067. https://doi.org/10.1016/0032-0633(91)90113-O

Comfort, R. H., Craven, P. D., \& Richards, P. G. (1995). A modified thermal conductivity for low density plasma magnetic flux tubes. Geophysical Research Letter, 22, 2457-2460. https://doi.org/10.1029/95GL02408 
Denton, R. E., Takahashi, K., Thomsen, M. F., Borovsky, J. E., Singer, H. J., Wang, Y., \& Reinisch, B. W. (2014). Evolution of mass density and O+ concentration at geostationary orbit during storm and quiet events. Journal of Geophysical Research: Space Physics, 119, 6417-6431. https://doi.org/10.1002/2014JA019888

Drob, D. P., Emmert, J. T., Crowley, G., Picone, J. M., Shepherd, G. G., Skinner, W., \& Vincent, R. A. (2008). An empirical model of the Earth's horizontal wind fields: HWM07. Journal of Geophysical Research, 113, A12304. https://doi.org/10.1029/2008JA013668

Drob, D. P., Emmert, J. T., Meriwether, J. W., Makela, J. J., Doornbos, E., Conde, M., \& Klenzing, J. H. (2015). An update to the Horizontal Wind Model (HWM): The quiet time thermosphere. Earth and Space Science, 2, 301-319. https://doi.org/10.1002/2014EA000089

Emmert, J. T., Drob, D. P., Shepherd, G. G., Hernandez, G., Jarvis, M. J., Meriwether, J. W., \& Tepley, C. A. (2008). DWM07 global empirical model of upper thermospheric storm-induced disturbance winds. Journal of Geophysical Research, 113, A11319. https://doi.org/10.1029/ 2008JA013541

Galvan, D. A., Moldwin, M. B., Sandel, B. R., \& Crowley, G. (2010). On the causes of plasmaspheric rotation variability: IMAGE EUV observations. Journal of Geophysical Research, 115, A01214. https://doi.org/10.1029/2009JA014321

Gulyaeva, T. L., Arikan, F., Stanislawska, I., \& Poustovalova, L. V. (2013). Symmetry and asymmetry of ionospheric weather at magnetic conjugate points for two midlatitude observatories. Advances in Space Research, 52(10), 1837-1844. https://doi.org/10.1016/j.asr.2012. 09.038

Hedin, A. E. (1987). MSIS-86 thermospheric model. Journal of Geophysical Research, 92, 4649-4662. https://doi.org/10.1029/ JA092iA05p04649

Hedin, A. E. (1991). Extension of the MSIS thermosphere model into the middle and lower atmosphere. Journal of Geophysical Research, 96, 1159-1172. https://doi.org/10.1029/90JA02125

Horwitz, J. L., Comfort, R. H., \& Chappell, C. R. (1990). A statistical characterization of plasmasphere density structure and boundary locations. Journal of Geophysical Research, 95, 7937-7947. https://doi.org/10.1029/JA095iA06p07937

Kalita, B. R., Hazarika, R., Kakoti, G., Bhuyan, P. K., Chakrabarty, D., Seemala, G. K., \& Roy, P. (2016). Conjugate hemisphere ionospheric response to the St. Patrick's Day storms of 2013 and 2015 in the $100^{\circ}$ E longitude sector. Journal of Geophysical Research: Space Physics, 121, 11,364-11,390. https://doi.org/10.1002/2016JA023119

Lawrence, D. J., Thomsen, M. F., Borovsky, J. E., \& McComas, D. J. (1999). Measurements of early and late time plasmasphere refilling as observed from geosynchronous orbit. Journal of Geophysical Research, 104, 14,691-14,704. https://doi.org/10.1029/1998JA900087

Lejosne, S., \& Mozer, F. S. (2018). Magnetic activity dependence of the electric drift below L = 3. Geophysical Research Letters, 45, 3775-3782. https://doi.org/10.1029/2018GL077873

Lejosne, S., \& Mozer, F. S. (2017). Subauroral Polarization Streams (SAPS) Duration as Determined From Van Allen Probe Successive Electric Drift Measurements. Geophysical Research Letters, 44, 9134-9141. https://doi.org/10.1002/2017GL074985

Liemohn, M. W., Khazanov, G. V., Moore, T. E., \& Guiter, S. M. (1997). Self-consistent superthermal electron effects on plasmaspheric refilling. Journal o Geophysical Research, 102, 7523-7536. https://doi.org/10.1029/96JA03962

Liu, L., Le, H., Chen, Y., He, M., Wan, W., \& Yue, X. (2011). Features of the middle- and low-latitude ionosphere during solar minimum as revealed from COSMIC radio occultation measurements. Journal of Geophysical Research, 116, A09307. https://doi.org/10.1029/ 2011JA016691

Marchaudon, A., \& Blelly, P. L. (2015). A new interhemispheric 16-moment model of the plasmasphere-ionosphere system: IPIM. Journal of Geophysical Research: Space Physics, 120, 5728-5745. https://doi.org/10.1002/2015JA021193

Marchaudon, A., Blelly, P. L., Grandin, M., Aikio, A., Kozlovsky, A., \& Virtanen, I. (2018). IPIM modeling of the ionospheric F2 layer depletion at high latitudes during a high-speed stream event. Journal of Geophysical Research: Space Physics, 123, 7051-7066. https:// doi.org/10.1029/2018JA025744

Menk, F. W., Ables, S. T., Grew, R. S., Clilverd, M. A., \& Sandel, B. R. (2012). The annual and longitudinal variations in plasmaspheric ion density. Journal of Geophysical Research, 117, A03215. https://doi.org/10.1029/2011JA017071

Nishida, A. (1966). Formation of plasmapause, or magnetospheric plasma knee, by the combined action of magnetospheric convection and plasma escape from the tail. Journal of Geophysical Research, 71, 5669-5679. https://doi.org/10.1029/JZ071i023p05669

Picone, J. M., Hedin, A. E., Drob, D. P., \& Aikin, A. C. (2002). NRLMSISE-00 empirical model of the atmosphere: Statistical comparisons and scientific issues. Journal of Geophysical Research, 107, 1468. https://doi.org/10.1029/2002JA009430

Reynolds, M. A., Ganguli, G., Su, Y. J., \& Thomsen, M. F. (2003). The local-time variation of the quiet plasmasphere: Geosynchronous observations and kinetic theory. Annales Geophysicae, 21, 2147-2154. https://doi.org/10.5194/angeo-21-2147-2003

Richards, P. G., Chang, T., \& Comfort, R. H. (2000). On the causes of the annual variation in the plasmaspheric electron density. Journal of Atmospheric and Solar-Terrestrial Physics, 62, 935-946. https://doi.org/10.1016/S1364-6826(00)00039-0

Sandel, B. R., Goldstein, J., Gallagher, D. L., \& Spasojevic, M. (2003). Extreme ultraviolet imager observations of the structure and dynamics of the plasmasphere. Space Science Reviews, 109, 25-46. https://doi.org/10.1023/B:SPAC.0000007511.47727.5b

Sandel, B. R., King, R. A., Forrester, W. T., Gallagher, D. L., Broadfoot, A. L., \& Curtis, C. C. (2001). Initial results from the IMAGE Extreme Ultraviolet Imager. Geophysical Research Letters, 28, 1439-1442. https://doi.org/10.1029/2001GL012885

Schulz, M. (2007). Magnetosphere. In Y. Kamide \& A. C.-L. Chian (Eds.), Handbook of the Solar-Terrestrial Environment (pp. 156-188). Berlin Heidelberg New York: Springer-Verlag. https://www.springer.com/gp/book/9783540463146

$\mathrm{Su}$, Y. J., Thomsen, M. F., Borovsky, J. E., \& Lawrence, D. J. (2001). A comprehensive survey of plasmasphere refilling at geosynchronous orbit. Journal of Geophysical Research, 106, 25,615-25,630. https://doi.org/10.1029/2000JA000441

Thomsen, M. F., McComas, D. J., Borovsky, J. E., \& Elphic, R. C. (1998). The magnetospheric trough. Washington DC American Geophysical Union Geophysical Monograph Series, 104, 355-369. https://doi.org/10.1029/GM104p0355

Timoçin, E., Ünal, İ., Tulunay, Y., \& Göker, Ü. D. (2018). The effect of geomagnetic activity changes on the ionospheric critical frequencies (foF2) at magnetic conjugate points. Advances in Space Research, 62(4), 821-828. https://doi.org/10.1016/j.asr.2018.05.035

Tu, J. N., Horwitz, J. L., Song, P., Huang, X. Q., Reinisch, B. W., \& Richards, P. G. (2003). Simulating plasmaspheric field-aligned density profiles measured with IMAGE/RPI: Effects of plasmasphere refilling and ion heating. Journal of Geophysical Research, 108, 1017. https:// doi.org/10.1029/2002JA009468 